\title{
ILCEA
}

Revue de l'Institut des langues et cultures

d'Europe, Amérique, Afrique, Asie et Australie

$24 \mid 2015$

Lire et écrire ensemble

\section{Lire ensemble}

\section{Christian Estrade}

\section{OpenEdition}

Journals

Édition électronique

URL : http://journals.openedition.org/ilcea/3521

DOI : 10.4000/ilcea.3521

ISSN : 2101-0609

Éditeur

UGA Éditions/Université Grenoble Alpes

Édition imprimée

ISBN : 978-2-84310-313-1

ISSN : 1639-6073

Référence électronique

Christian Estrade, «Lire ensemble », ILCEA [En ligne], 24 | 2015, mis en ligne le 15 octobre 2015 consulté le 24 septembre 2020. URL : http://journals.openedition.org/ilcea/3521 ; DOI : https:// doi.org/10.4000/ilcea.3521

Ce document a été généré automatiquement le 24 septembre 2020.

(C) ILCEA 


\title{
Lire ensemble
}

\author{
Christian Estrade
}

1 Je ne sais pas vraiment où me situer pour présenter cet hommage à Michel Lafon écrit à plusieurs voix, comment présenter ce concert dédié à son œuvre. L'écriture et l'édition d'un tel ouvrage sont tristement liées à la mort, bien qu'une énergie très sensible parcourt ce recueil - mieux que mélange - car les contributions parlent toutes de lecture, de collaboration, d'amitié, de co-écriture et de partage.

2 Ce numéro de la revue ILCEA autour de l'œuvre de Michel Lafon n'a pas pris la forme d'un je me souviens. Cet hommage a eu lieu ailleurs, à Paris, organisé par son ami Benoît Peeters dans un appartement parisien le 12 décembre 2014, peu après sa disparition. Celui-ci veut rendre hommage à l'universitaire, au professeur, au collègue, au maître qu'il a été pour un grand nombre, sans pour autant omettre le souvenir, à l'instar de ses amis argentins. César Aira revient dans « Todas las primeras veces, o sea siempre » sur une amitié qui n'a de cesse de renaitre, Eduardo Berti se souvient d'une amitié qui prend forme au fil des ans, Luis Chitarroni dans « Dos veces el mar» dialogue avec son ami aux mille facettes et Noé Jitrik partage un texte allégorique sur l'amitié et l'écriture.

3 Il y a dans l'œuvre de Michel Lafon une formidable cohésion qui, il est vrai, gravite autour de Jorge Luis Borges peut-être, comme l'affirme Milagros Ezquerro, par simple addiction. L'auteur de Ficciones est immanquablement au centre des ouvrages les plus importants de Michel. Borges ou la réécriture publiée au Seuil en 1990 est aujourd'hui une pièce majeure dans les études borgésiennes. Une pièce où Michel établit la réécriture comme le nœud conceptuel, de la poésie à l'essai, qui sous-tend toute l'œuvre de Jorge Luis Borges, mais où il consolide également le concept de réécriture qui fera florès aux côtés de notions contemporaines comme celle d'intertextualité de Gérard Genette. Ce concept de réécriture excède l'œuvre de Borges pour étendre son emprise sur toute la littérature $\mathrm{du} \mathrm{xx}^{\mathrm{e}}$ siècle. Elle permet ici à Julien Roger de postuler la notion de réécrivain ou d'intervenir subtilement dans l'écriture d'un essai comme l'avoue Daniel Attala au terme de sa contribution « El factor humano ».

De la même manière, Nous est un autre, écrit à quatre mains avec Benoît Peeters - qui nous dévoile ici dans "Écrire ensemble " l'architecture du deuxième volet plus 
théorique de leur recherche commune - explore l'écriture en collaboration en partant notamment de celle de Borges et Bioy Casares, vers une littérature sans frontières, pour poser une profonde réflexion sur la notion sacralisée d'auteur en littérature.

Son dernier travail sur Borges représente si ce n'est une refondation tout du moins une nouvelle perspective dans sa recherche. Michel engage alors un tournant génétique qui lui permet de donner vie aux variantes de deux manuscrits majeurs de Borges et de lire les feuillets palpitants des cuentos « El Sur » et « Tlön, Uqbar, Orbis Tertius », comme les partitions de l'univers borgésien qui a tant fasciné lecteurs et chercheurs. La génétique, ou lire la forme de la création, fascinait Michel qui se prenait à lire les manuscrits comme de véritables pierres de Rosette.

6 À Borges, Michel revenait sans cesse, mais pour aller toujours plus loin, comme dans son dernier projet que nous publions ici, «Éditer et lire Borges en France (1939-1970) Pour une poétique de la réception", qui devait aboutir à une œuvre monumentale : Traduire Borges. Il s'agit d'un projet présenté à l'IUF qui n'a pas pu voir le jour, organisé autour d'un enchainement notionnel imposant : éditer, traduire, diffuser, lire, critiquer, interpréter; et qui regroupait le résultat de ses longues années de recherche en traductologie. N'oublions pas cependant de rappeler que si l'œuvre de l'auteur de Ficciones a été pour Michel un espace de recherche et de réflexion sur la littérature, il a été également un espace de création avec la publication d'Une vie de Pierre Ménard en 2008, lauréat du Prix Valery Larbaud l'année suivante.

7 Ces lignes de recherche ouvertes ou explorées par Michel, la réécriture et l'écriture en collaboration, la génétique et la traductologie, sans oublier sa passion pour la bande dessinée, donnent lieu à de nouvelles perspectives qui se déclinent en de croisements productifs. Ce sont ces croisements qui opèrent dans des contributions comme celles de Cécile Meynard sur la fonction des dessins dans les manuscrits de Stendhal au service des descriptions ; de Claire Latxague qui propose une lecture sur la collaboration dans un roman graphique argentin contemporain entre un dessinateur et trois scénaristes; mais également dans la contribution de Juanita Cifuentes où la danse et la poésie se croisent dans l'œuvre de la poétesse Susana Thénon et dans celle de Margarita Remón qui propose de reformuler le genre essai, avec une formulation originale, lire en compagnie, en parcourant la bibliothèque argentine de l'écrivain mexicain Juan Villoro. D'autres collaborateurs explorent dans leurs lectures en hommage à Michel diverses formes d'amitié dans la littérature. Corinne Ferrero dialogue avec un roman de Daniel Guebel qui est un véritable tribut à ses mentors, deux écrivains secrets des lettres argentines contemporaines, et Carla Fernandez propose de lire Une vie de Pierre Ménard comme un roman motivé et imprégné d'amitié.

8 Nous ne devrions cependant pas nous limiter à lire l'héritage de Michel dans ces œuvres autour de Borges, son travail pour la diffusion de la littérature argentine en France ces vingt dernières années a été central. Dans son rôle de professeur il a dirigé vingt thèses de doctorat et deux HDR, portant pour l'essentiel sur les littératures contemporaines du Rio de la Plata, qui couvrent la poésie, l'essai, les formes brèves, la bande dessinée, l'écriture en collaboration. Aussi, il reste en France le fondateur de la seule chaire de littérature argentine et en Argentine il était depuis 2010, membre correspondant en France de l'Académie argentine des lettres. Hormis son amitié et ses traductions des novelitas de César Aira, Michel était en relation permanente avec les écrivains argentins contemporains, avec ceux de la génération de Ricardo Piglia, avec ceux de la génération Babel, mais également avec les plus jeunes, les primo-romanciers, 
dont il recevait de manière systématique les manuscrits et lui demandaient souvent conseil. Michel a été un passeur infatigable des lettres argentines depuis les années 1990.

9 Caprice du préfacier, je voudrais rapporter deux autres textes qui nous rappellent que Michel n'écrivait pas que de magnum opus à partir de l'œuvre de Borges. Le premier est à la croisée de la collaboration et de sa vie de créateur qui ne se limite pas à son Ménard - une nouvelle forme de réinventer son dévouement à l'auteur de Ficciones, mais de l'autre côté du miroir - pour rejoindre son autre passion qu'était la bande dessinée avec la publication de La conjuration de Baal, un épisode d'Alix dont il a été le scénariste. Preuve que pour ce chercheur invétéré qui revenait toujours à Borges par un autre chemin sa vie d'écrivain ne se limitait pas à celle de réécrivain-romancier. Le deuxième est un essai bref sur la forme brève, le cuento, c'est-à-dire un genre qui dans la tradition hispanique occupe une place très importante, qui plus est dans la littérature hispanoaméricaine où il n'a de cesse d'être théorisé. Dans ce texte « Pour une poétique de la forme brève ", Michel propose une thèse incontournable et nous pourrions dire spectaculaire qui tient sur dix pages mais également sur une seule phrase avec la clarté qu'il cultivait et exigeait de ses doctorants: "La forme brève n'est pas une forme solitaire, mais au contraire une forme solidaire». Pourraient enrichir cet exemple d'autres essais sur la mise en recueil, sur le journal intime ou le romanesque.

Ce tissage entre la vie de chercheur et la vie d'écrivain où tout semble circuler avec une cohérence insoupçonnée avait un espace de résonance dans l'enseignement. Les séminaires qu'il animait dans l'axe "Rio de la Plata » - les formes brèves, la sciencefiction, l'essai - constituaient un espace de partage et d'échanges. Son dernier séminaire sur le romanesque, thématique qui le fascinait, donnait lieu à des séances foisonnantes. Il y a là sans doute une notion qui définit le mieux Michel, qui pouvait passer des heures à regarder vignette après vignette des planches de Peyo, siffloter en conduisant des tangos ou reprendre le programme du prochain concert de son pianiste préféré Alexandre Tharaud, écouter des séminaires de Barthes sur son disc-man, réviser une de ses collections, par exemple celle de pièces antiques, admirer les couvertures des romans de Fantômas. Il ne nous a pas complètement quittés puisqu'il nous laisse un sens de l'amitié intellectuelle et des outils pour penser le littéraire, pour écrire ensemble.

\section{AUTEUR \\ CHRISTIAN ESTRADE}

IRIEC, université Toulouse - Jean Jaurès 